\title{
A Possible Origin for the LFQPO Types In Microquasars
}

\section{Peggy Varniere}

AstroParticule \& Cosmologie (APC), UMR 7164, Université Paris Diderot, 10 rue Alice Domon et Lńeonie Duquet, 75205 Paris Cedex 13, France

E-mail: varnieredapc.univ-paris7.fr

\section{J. Rodriguez}

CEA Saclay, DSM/IRFU/SAp, Laboratoire AIM F-91191 Gif sur Yvette France

E-mail: jerome.rodriguez@cea.fr

\section{Tagger}

LPCE, Orleans et AstroParticule \& Cosmologie (APC), UMR 7164, Université Paris Diderot, 10 rue Alice Domon et Lńeonie Duquet, 75205 Paris Cedex 13, France

E-mail: Michel.Taggerecnrs-orleans.fr

We have presented the Accretion-Ejection Instability (AEI) as an explanation for the LowFrequency Quasi-Periodic Oscillation in its most usual form, the C-type. Recently we have shown that relativistic effects result in another flavor of the AEI, the Relativistic AEI, when the disk gets close to its last stable orbit. Here we will focus on the consequences on the LFQPO and how this can be used to explain the less frequent A- and B-type of LFQPO.

VII Microquasar Workshop: Microquasars and Beyond September 1-5 2008

Foca, Izmir, Turkey 


\section{Introduction}

The Accretion-Ejection Instability (AEI) was first presented as a possible explanation for the Low-Frequency Quasi-Periodic Oscillation (LFQPO) in Tagger \& Pellat 99 and has since been shown to be a good candidate to explain the LFQPO in its most usual form, the type C LFQPO. First of all it is an instability, therefore it does not require external excitation, but grows naturally to a high amplitude. It is also able to account for the following observational characteristics:

- the rotation frequency of the dominant $m=1$ mode ( of the order of a tenth of the keplerian frequency at the inner edge) is consistent with the LFQPO frequency.

- based on zeroth order approximation of variations in disk properties at the location of the spiral wave, the AEI partially reproduces the observed X-ray flux modulation.

- non-linear simulations show that the rotating pattern of the AEI remains nearly steady, and thus is able to account for persistent LFQPOs.

- the AEI transfers energy and angular momentum toward the corona by Alfven Waves, thus providing a supply of Poynting flux that may produce the compact jet, often observed in the hard state.

- by including General Relativity through the existence of a last stable orbit and orbital velocity profile, the relativistc AEI can explain the observed turnover in the correlation between the color radius (= inner disk radius, as determined by the spectral fits) and the LFQPO frequency.

It was recently shown that when the inner edge of the disk is close to its last stable orbit the disk can present both the Rossby Wave Instability (possibly at the origin of the HFQPO see Tagger \& Varniere 2006) and the relativistic flavor of the AEI active in the disk (see Varniere et al. this proceeding for details about the classification). In that case we would have HFQPO and LFQPO present in the disk. Here we study the possibility that the relativistic AEI is at the origin of the Aand B-type LFQPOs.

\section{The observed LFQPO types}

During the 1998 outburst of XTE J1550-564 several types of LFQPOs were observed and labeled A, B and C (Remillard et al. 2002) depending on their characteristics. The C-type is the one occurring the most regularly and in every LFQPO source. It is the one that we proposed to be an expression of the Accretion-Ejection Instability dominating the inner region of the disk [4] while B and A types were, up to recently, only observed in outbursting sources (in 2007, the first B-type LFQPO was observed in GRS $1915+105$ by Soleri et al.).

From the beginning, A- and B-types LFQPO were associated with the presence of HFQPOs and the recent observation of a B type LFQPO in GRS 1915+105 happened while HFQPOs were also detected, strengthening that association. Also when the HFQPO appears the LFQPO frequency tends to decrease and their Q-values are anti-correlated, hinting toward a competition mechanism.

\section{Relatvistic AEI + RWI : LFQPO type and HFQPO}

We recently proposed a new classification of microquasar states based on the action of three distinct instabilities (MRI, AEI and RWI, see Varniere et al. this proceeding for details). Here we 


\begin{tabular}{lccc}
\hline properties & type A & type B & type C \\
\hline frequency (Hz) & $\sim 6$ & $\sim 6$ & $0.1-10$ \\
Amplitude (\%rms) & $3-4$ & $\sim 4$ & $3-16$ \\
Q $v / F W H M$ & $\sim 2-4$ & $\sim 4$ & $>10$ \\
Phase Lag (rad) & -0.6 to -1.4 & 0 to 0.4 & 0.05 to -0.4 \\
sub-harmonic & $\ldots$ & soft & soft \\
1st harmonic & soft & soft & hard \\
coherence & $<0.5$ & $\sim 1$ & $\sim 0.9$ \\
HFQPO & $4 / 4$ & $6 / 9$ & $5 / 51$ \\
Noise & weak red & weak red & strong flat top \\
\hline
\end{tabular}

Table 1: Observational definition of the different types of LFQPO. It is a summary of the papers by [1] and [?] based on the microquasars XTE J1550-564 .

are mostly interested in the manner in which the AEI is modified by relativistic effects due to the fact that the inner edge of the disk is at the last stable orbit. We ran a 2D MHD disk simulation with initial conditions such as to obtain the RWI at the inner edge of the disk and the AEI further away in the disk.

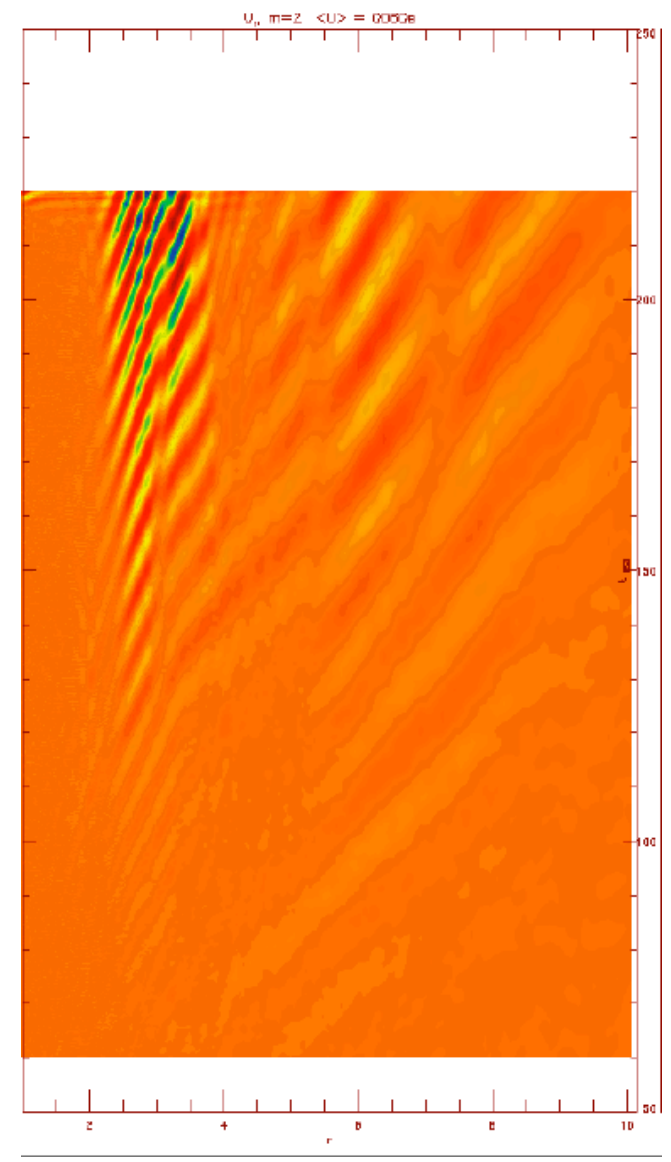

This figure on the left shows the evolution, as a function of radius (abscissa) and time (ordinate) of the $m=2$ mode in the disk. It clearly shows fluctuations (the oblique patterns) at two different frequencies. They correspond to the $m=2$ RWI close to the last stable orbit (about $1.3 r_{L S O}$ in that particular simulation) and the $m=2$ mode of the AEI occuring at a higher radius $\left(\sim 5 r_{L S O}\right)$.

When comparing it with the observations of the three LFQPOs types we thus propose to associate the different types with a different mode of the AEI

- type C LFQPO is an expression of the AEI alone, mainly the $m=1$ mode

-type B LFQPO is an expression of the Relativistic-AEI with the $m=2$ mode dominant while the $m=1$ is still present.

- type A LFQPO is also an expression of the Relativistic-AEI but with the $m=3$ mode dominating, with still modes $m=2$ and $m=1$ present. 


\section{Comparison with observation}

In order to compare observation we take the case of XTE J1550-564 which exhibits these different types with regularity.

\section{1 the case of XTE $\mathbf{J} 1550-564$}

Frequency of the A- and B-types LFQPOs of the order of $6 \mathrm{~Hz}$ :

The first striking feature of the A- and B-types LFQPO is the small range of frequency they have compared to the C-type. This is simply explained by the criteria to obtain a state with the relativistic AEI. The inner edge of the disk needs to be very close to the last stable orbit.In that zone the LFQPO frequency varies, at most, by a factor of two. Within that framework, the A- and B-types LFQPO are not allowed to change in frequency as much as the C-type.

\section{Link with the mode of the HFQPO:}

In order to enter in the Relativistic AEI regime, we need to have the inner edge of the disk close to the last stable orbit, Therefore the RWI also becomes active, explaining the link between the HFQPO mode and LFQPOs of A- and B-types.

\section{(Sub)-Harmonic content of the LFQPO :}

A striking feature of the LFQPOs during that outburst is the presence of sub-harmonics. Within the AEI-RWI state, those "harmonics" and "sub-harmonics" of the mode with the higher rms, come from the different AEI modes. These modes are not exactly in harmonic relation but close enough. In the case of the AEI alone the mode $m=1$ is the dominant one, meaning the one with the higher amplitude. The other modes observed are much weaker and given their weak quality factor may appear as a set of harmonics. But in the AEI-RWI state the dominant mode of the AEI is not the $m=1$ but a is a higher mode, for example the $m=2$ of the AEI is dominant in the presence of the $m=2$ RWI. In that case the weaker $m=1$ mode would appear as a sub-harmonic of the dominant mode.

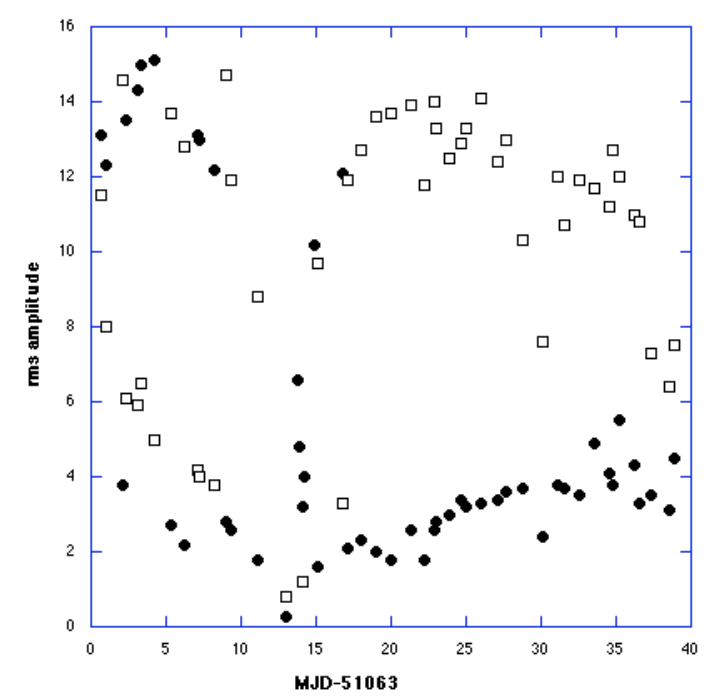

This would also explain the difference in the coherence and lag behavior, since they are distinct modes of the same instability and not harmonics.

The figure on left shows evolution of the rms amplitude of the two lower frequency QPOs observed during the 1998 outburst of XTE J1550-564 as function of time. The circles represent the lowest frequency and the square the LFQPO about twice that frequency. Both LFQPOs stay present through the outburst but the dominant one changes from the lowest frequency to about twice that frequency. 
This is coherent with an initially dominant $m=1$ mode which is slowly replaced by the $m=2$ mode as the disk enters the AEI+RWI state; another point toward that interpretation is that as the transition between the two modes occurs the $v=183 \mathrm{~Hz}$ HFQPO begins to be detectable reenforcing the idea that the disk is in the $\{$ AEI-RWI $\}$ state.

\subsection{Other Objects}

The case of GRO J1655-40:

During the outburst of 96-97, the LFQPO and the spectral parameter had peculiar correlations which were explained using the AEI as a model for the LFQPO by assuming that the inner radius of the disk was close to its last stable orbit during most of the outburst. HFQPOs were actually observed during that outburst which is coherent with the disk having both the RWI and the relativistic AEI present in the disk. During this outburst the LFQPO is shown to have an "almost" first-harmonic present (almost because the two frequencies are not exactly in an harmonic relationship). We need to explore the presence (or not) of B- or A-type LFQPO. It is therefore necessary to re-analyse the data for the LFQPO and check the amplitude and frequencies present.

The case of H1743-32:

The link between LFQPO type and HFQPO can be used as a mean to detect HFQPOs. This was done using phenomenological aspects of the PDS when HFQPOs are observed. In 2005 Homan et al. discovered 2 HFQPOs in H1743-322 and observed A- and B-type LFQPOs. Later Remillard et al. 2006, grouped the 130 observations in four groups depending on the characteristics of the LFQPOs and found HFQPOs in two of them, which exhibit the characteristics of a state with the Relativistic AEI + RWI active.

\section{Conclusion}

We have proposed to associate the three types of LFQPO with the position of the inner disk radius in a fully magnetized disk: when it approaches the last stable orbit, it allows the disk to present both the AEI, at the origin of the LFQPO, and the RWI at the origin of the HFQPO. The occurrence of both instabilities is a good candidate to explain the behaviour of the A- and B-type of the LFQPO such as their quasi-stable-frequency, their (sub)-harmonic content or the relationship with the HFQPO, while keeping the AEI alone for the C-type, when the inner radius moves away from the LSO

\section{References}

[1] Remillard et al., 2002, ApJ, 564, 962.

[2] Remillard, et al., 2006, ApJ, 637, 1002.

[3] Soleri, et al., 2007, MNRAS, 383, 1089.

[4] Tagger \& Pellat, 1999, A\&A

[5] Tagger, M. \& Varniere, P., 2006, ApJ, 652, 1457. 